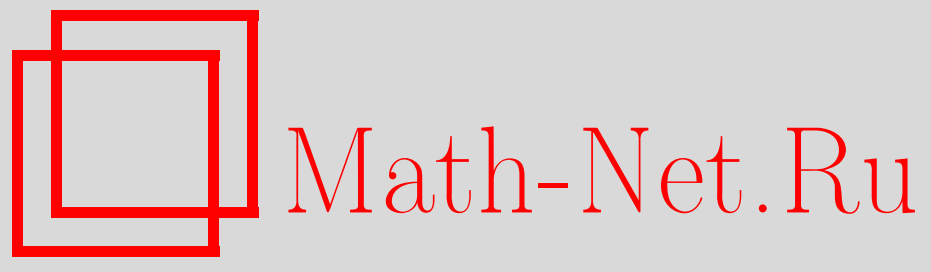

Е. М. Коленова, А. М. Себельдин, Об изоморфности абелевой группы своей группе эндоморфизмов, Матем. заметки, 2006, том 80, выпуск 4, 536-545

DOI: https://doi.org/10.4213/mzm2846

Использование Общероссийского математического портала Math-Net.Ru подразумевает, что вы прочитали и согласны с пользовательским соглашением http: //www . mathnet.ru/rus/agreement

Параметры загрузки:

IP: 54.174 .149 .18

26 апреля 2023 г., 15:32:47

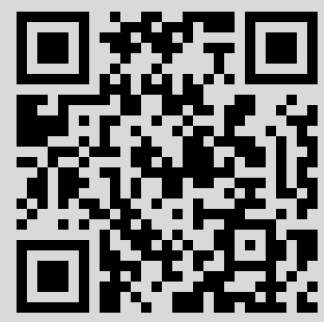




\section{ОБ ИЗОМОРФНОСТИ АБЕЛЕВОЙ ГРУППЫ СВОЕЙ ГРУППЕ ЭНДОМОРФИЗМОВ}

\section{Е. М. Коленова, А. М. Себельдин}

В работе получены необходимые и достаточные условия изоморфности абелевой группы $A$ и группы эндоморфизмов $\operatorname{End}(A)$. Рассмотрены классы периодических абелевых групп, делимых абелевых групп, нередуцированных абелевых групп и редуцированных алгебраически компактных абелевых групп. Для некоторых классов абелевых групп в настоящей работе решена задача изоморфности группы и ее группы эндоморфизмов при условии, что сама группа эндоморфизмов этим свойством обладает.

Библиография: 5 названий.

Важной задачей теории абелевых групп является изучение связей между абелевой группой и ее группой эндоморфизмов. Естественным образом возникает вопрос, при каких условиях абелева группа и ее группа эндоморфизмов изоморфны (см. [1; проблема 31], [2; проблемы 40, 45]). Ответ на этот вопрос получен Себельдиным в классах вполне разложимых абелевых групп [3], векторных групп [4]. В настоящей работе задача изоморфности абелевой группы $A$ и ее группы эндоморфизмов $\operatorname{End}(A)$ решена в классах периодических абелевых групп (теорема 1), делимых абелевых групп (теорема 2), нередуцированных абелевых групп (теорема 3), редуцированных алгебраически компактных абелевых групп (теорема 4). В каждом случае получены условия, необходимые и достаточные для изоморфизма $\operatorname{End}(A) \cong A$. Для некоторых классов абелевых групп в настоящей работе решена задача изоморфности группы и ее группы эндоморфизмов при условии, что сама группа эндоморфизмов обладает этим свойством.

Все группы, рассматриваемые в работе абелевы. Для простоты изложения определим некоторые используемые в работе понятия и обозначения:

$\mathbb{P}$ - множество всех простых чисел;

$\mathbb{N}^{*}=\mathbb{N} \cup 0$, где $\mathbb{N}$ - множество всех натуральных чисел; $P \subset \mathbb{N} \subset \mathbb{N}^{*}$;

$T(A)$ - периодическая часть группы $A$;

$r(A)$ - ранг группы $A$;

$r(0, A)$ - ранг без кручения группы $A$;

$S(A)$ - множество всех тех простых $p$, для которых периодическая $p$-компонента группы $A$ ненулевая;

$P(A)$ - множество всех тех простых $p$, для которых $p$-адическая компонента редуцированной алгебраически компактной группы $A$ ненулевая.

(C) Е. М. КоленовА, А. М. СЕБельдин, 2006 
Пусть $D(A)$ - делимая часть группы $A$, тогда $A=D(A) \oplus R(A)$; назовем дополнительное прямое слагаемое $R(A)$, определенное с точностью до изоморфизма, $p е д y$ иированной частъю группы $A$.

Делимая часть $D(A)$, в свою очередь, также разлагается:

$$
D(A)=D t(A) \oplus D f(A), \quad \text { где } \quad D t(A)=T(D(A)),
$$

а дополнительное прямое слагаемое $D f(A)$, определенное с точностью до изоморфизма, будем называть частью без кручения делимой группы $D(A)$. Далее везде, где будет удобно и не вызовет недоразумений, вместо $D(A), D f(A)$ и $\operatorname{Dt}(A)$, будем использовать соответственно обозначения $D, D f$ и $D t$.

Остальные обозначения соответствуют [1] или вводятся по ходу изложения.

Для периодических групп ответ на поставленный вопрос получить несложно (см. замечание к проблеме 31 книги [1]). А именно, имеет место

Теорема 1. Периодическая группа изоморфна своей группе эндоморфизмов тогда и только тогда, когда она циклическал.

ДокАЗАТЕЛЬСтво. Пусть вначале $A-p$-группа и имеет место изоморфизм $\operatorname{End}(A) \cong A$, тогда $A-$ конечная группа $[1 ;$ с. 215$]$ и поэтому является прямой суммой конечного числа циклических групп [1; с. 95]. Таким образом,

$$
A \cong \bigoplus_{k \in K} \mathbb{Z}\left(p^{k}\right)
$$

где $K$ - некоторое конечное семейство натуральных чисел (не обязательно различных). Покажем, что $|K|=1$. На самом деле, с одной стороны

$$
\operatorname{End}(A) \cong \operatorname{End}\left(\bigoplus_{k \in K} \mathbb{Z}\left(p^{k}\right)\right) \cong \bigoplus_{k^{\prime}, k^{\prime \prime} \in K} \operatorname{Hom}\left(\mathbb{Z}\left(p^{k^{\prime}}\right), \mathbb{Z}\left(p^{k^{\prime \prime}}\right)\right) \cong \bigoplus_{k^{*} \in K^{*}} \mathbb{Z}\left(p^{k^{*}}\right),
$$

где $k^{*}=\min \left\{k^{\prime}, k^{\prime \prime}\right\}$, а с другой стороны,

$$
\operatorname{End}(A) \cong A \cong \bigoplus_{k \in K} \mathbb{Z}\left(p^{k}\right)
$$

Откуда следует, что $|K|=\left|K^{*}\right|$. А это возможно, если только $|K|=1$. Итак, $A \cong \mathbb{Z}\left(p^{k}\right)$, т.е. является конечной циклической группой.

Пусть теперь $A$ - периодическая группа и $\operatorname{End}(A) \cong A$. Известно, что периодическая группа может быть разложена, притом единственным способом, в прямую сумму примарных групп $A p$, относящихся к различным простым числам [1; с. 55], поэтому $A=\bigoplus_{p \in S(A)} A p$. Имеем [1; с. 214]

$$
\operatorname{End}(A) \cong \operatorname{End}\left(\bigoplus_{p \in S(A)} A p\right) \cong \prod_{p \in S(A)} \operatorname{End}(A p)
$$

и, следовательно,

$$
\operatorname{End}(A) \cong \bigoplus_{p \in S(A)} A p
$$


Следовательно, $S(A)$ - конечное множество и для любого $p$ из $S(A)$ выполняется изоморфизм $\operatorname{End}(A p) \cong A p$. Но это возможно лишь только в том случае, когда каждая $p$-компонента $A p$ группы $A$ является циклической группой, т.е. $A$ является циклической группой.

Обратно, если $A$ - циклическая группа, то, очевидно, имеет место изоморфизм $\operatorname{End}(A) \cong A$.

Случай делимых групп также несложен. А именно, имеет место

ТЕорема 2. Делимая группа изоморфна своей группе эндоморфизмов в том и только том случае, когда она изоморфна аддитивной группе рациональных чисел $\mathbb{Q}$.

ДокАЗАтеЛЬство. Достаточность очевидна.

Необходимость. Пусть $A$ - делимая группа и $\operatorname{End}(A) \cong A$. Так как группа $A$ делимая, то $\operatorname{End}(A)$ - группа без кручения [1; с. 213$]$, а поскольку $\operatorname{End}(A) \cong A$, то и группа $A$ - группа без кручения. Получаем, что $A$ - делимая группа без кручения, поэтому она является прямой суммой групп, изоморфных группе рациональных чисел:

$$
A \cong \bigoplus_{r(A)} \mathbb{Q}
$$

Имеем, с одной стороны,

$$
\begin{aligned}
\operatorname{End}(A) & \cong \operatorname{End}\left(\bigoplus_{r(A)} \mathbb{Q}\right)=\operatorname{Hom}\left(\bigoplus_{r(A)} \mathbb{Q}, \bigoplus_{r(A)} \mathbb{Q}\right) \\
& \cong \prod_{r(A)} \operatorname{Hom}\left(\mathbb{Q}, \bigoplus_{r(A)} \mathbb{Q}\right) \cong \prod_{r(A)}\left[\bigoplus_{r(A)} \mathbb{Q}\right]
\end{aligned}
$$

С другой стороны, по условию

$$
\operatorname{End}(A) \cong A \cong \bigoplus_{r(A)} \mathbb{Q} .
$$

Следовательно, $r(A)=1$ и $A \cong \mathbb{Q}$.

ТЕорема 3. Нередуцированная группа изоморфна своей группе эндоморфизмов тогда и только тогда, когда ее делимая часть изоморфна группе рачиональных чисел $\mathbb{Q}$, а редуцированная часть либо равна нулю, либо конечная ииклическал.

ДокАЗАтЕльство. Пусть $A=D(A) \oplus R(A)$ - нередуцированная группа, т.е. $D(A)$ отлична от нуля. В силу теоремы 2 достаточно рассмотреть случай, когда $R(A)$ отлична от нуля.

Достаточность. Если $D(A) \cong \mathbb{Q}$ и $R(A) \cong \mathbb{Z}(n)$, то

$$
\operatorname{End}(A) \cong \operatorname{End}(\mathbb{Q}) \oplus \operatorname{Hom}(\mathbb{Q}, \mathbb{Z}(n)) \oplus \operatorname{End}(\mathbb{Z}(n)) \oplus \operatorname{Hom}(\mathbb{Z}(n), \mathbb{Q}) .
$$

А так как $\operatorname{Hom}(\mathbb{Q}, \mathbb{Z}(n))$ и $\operatorname{Hom}(\mathbb{Z}(n), \mathbb{Q})$ - нулевые группы, то получаем, что $\operatorname{End}(A)$ $\cong \mathbb{Q} \oplus \mathbb{Z}(n)$, т.е. $\operatorname{End}(A) \cong A$.

Необходимость. Пусть для группы $A=D(A) \oplus R(A) \quad(D(A) \neq 0$ и $R(A) \neq 0)$ выполняется изоморфизм $\operatorname{End}(A) \cong A$. Тогда

$$
\operatorname{End}(A) \cong \operatorname{Hom}(D f(A), A) \oplus \operatorname{Hom}(D t(A), A) \oplus \operatorname{Hom}(R(A), A) .
$$


Так как группа $D f(A)$ делимая без кручения, то

$$
D f(A) \cong \bigoplus_{r(0, D(A))} \mathbb{Q}
$$

и

$$
\operatorname{Hom}(D f(A), A) \cong \prod_{r(0, D(A))} \operatorname{Hom}(\mathbb{Q}, A) .
$$

Заметим, что ранг без кручения $r(0, D(A))$ может быть только конечным, иначе равенство $|D f(\operatorname{End}(A))|=|D f(A)|$ не выполняется. Имеем

$$
\operatorname{Hom}(D f(A), A) \cong \bigoplus_{r} \mathbb{Q} \oplus \operatorname{Hom}(\operatorname{Df}(A), \operatorname{Dt}(A)),
$$

где $r=[r(0, D(A))]^{2}$, а группа $\operatorname{Hom}(D f(A), A)$ делимая без кручения [1; с. 213]. А поскольку $\operatorname{End}(A) \cong A$, то

$$
\bigoplus_{r} \mathbb{Q} \oplus \operatorname{Hom}(D f(A), D t(A)) \cong \bigoplus_{r(0, D(A))} \mathbb{Q},
$$

откуда следует, что $r(0, D(A)) \leqslant 1$ и $\operatorname{Hom}(D f(A), D t(A))=0$.

Таким образом, возможны только два случая:

$$
r(0, D(A))=1 \quad \text { или } \quad r(0, D(A))=0 .
$$

Случай 1. Пусть ранг без кручения $r(0, D(A))$ группы $D(A)$ равен 1 , т.е. $D f(A) \cong$ $\mathbb{Q}$. Тогда из изоморфизма

$$
\operatorname{Hom}(D f(A), \operatorname{Dt}(A)) \cong \operatorname{Hom}(Q, D t(A))=0,
$$

следует, что $D t(A)=0$. В этом случае делимая часть $D(A)$ группы $A$ изоморфна $\mathbb{Q}$, и тогда

$$
A \cong \mathbb{Q} \oplus R(A) \quad \text { и } \quad \operatorname{End}(A) \cong \mathbb{Q} \oplus \operatorname{Hom}(R(A), A) .
$$

Рассмотрим группу $\operatorname{Hom}(R(A), \mathbb{Q})$, которая, с одной стороны, является делимой группой [1; с. 213], а с другой стороны, - подгруппой группы $\operatorname{Hom}(R(A), A)$. Однако $D(\operatorname{End}(A)) \cong D(A) \cong \mathbb{Q}$, значит, $\operatorname{Hom}(R(A), \mathbb{Q})=0$, и поэтому ранг без кручения $r(0, R(A))$ группы $R(A)$ равен нулю, т.е. $R(A)$ - периодическая группа. Следовательно,

$$
\operatorname{Hom}(R(A), A) \cong \operatorname{Hom}(R(A), \mathbb{Q} \oplus R(A)) \cong \operatorname{End}(R(A)) \cong R(\operatorname{End}(A)) \cong R(A) .
$$

Теперь, применяя теорему 1 для периодической группы $R(A)$, делаем вывод, что она является циклической.

Итак, в случае, когда $D f(A) \neq 0$ и $R(A) \neq 0$, изоморфизм $A \cong \operatorname{End}(A)$ влечет $A \cong \mathbb{Q} \oplus \mathbb{Z}(n)$.

Случай 2. Пусть теперь ранг без кручения $r(0, D(A))$ группы $D(A)$ равен нулю, т.е. $D(A)$ - ненулевая периодическая группа. Покажем, что в этом случае группа $A$ не изоморфна своей группе эндоморфизмов $\operatorname{End}(A)$ (напомним, что $R(A) \neq 0)$. Далее, в доказательстве этой теоремы положим $D=D(A)$. 
Так как группа $D$ делимая периодическая, то

$$
D \cong \bigoplus_{p \in S(D)}\left(\bigoplus_{r(p, D)} \mathbb{Z}\left(p^{\infty}\right)\right)=\bigoplus_{p \in S(D)} D p .
$$

Группа $\operatorname{End}(D)$ - редуцированная алгебраически компактная группа [1; с. 226]. Следовательно, в силу изоморфизма

$$
D \oplus R(A)=A \cong \operatorname{End}(A)=\operatorname{Hom}(R(A), D) \oplus \operatorname{End}(D) \oplus \operatorname{End}(R(A))
$$

группа $\operatorname{Hom}(R(A), D)$ отлична от нуля и содержит в качестве прямого слагаемого группу, изоморфную $D$. Пусть

$$
\operatorname{Hom}(R(A), D) \cong D \oplus H_{1} .
$$

Тогда

$$
R(A) \cong H_{1} \oplus \operatorname{End}(D) \oplus \operatorname{End}(R(A)) .
$$

Ясно [1; с. 233], что для любого $p$ из $S(D), \operatorname{Hom}(R(A), D p) \neq 0$, где

$$
D p=\bigoplus_{r(p, D)} \mathbb{Z}\left(p^{\infty}\right)
$$

- $p$-компонента группы $D$. Зафиксируем $p \in S(D)$, и пусть $B=\bigoplus_{n \in \mathbb{N}^{*}} B_{n}-$ $p$-базисная подгруппа группы $R(A)$, где $B_{0}=\bigoplus_{m(0)} \mathbb{Z}, B_{n}=\bigoplus_{m(n)} \mathbb{Z}\left(p^{n}\right), m(0)$ и $m(n)$ - некоторые кардиналы, $n \in \mathbb{N}$. Согласно [1; с. 233] $m(0) \neq 0$ и

$$
\operatorname{Hom}\left(B_{0}, D p\right) \cong \prod_{m(0)} D p
$$

- делимое прямое слагаемое группы $\operatorname{Hom}(R(A), D p)$ и, следовательно, группы $A$, точнее, группы $D p$ и, разумеется, с точностью до изоморфизма. Ясно [1; с. 233], что $m(0)$ - конечный кардинал (иначе мощность группы $\prod_{m(0)} D p$ больше мощности группы $D p)$.

Предположим сначала, что $p$-ранг $r(p, D)$ группы $D$ бесконечен, и пусть $\operatorname{End}(A) \cong$ $A$. В этом случае в группе $\operatorname{End}(D)$, а следовательно, и в $R(A)$ можно выделить в качестве прямых слагаемых $m(0)+1$ прямое слагаемое, изоморфное аддитивной группе $\mathbb{J}_{p}$ целых $p$-адических чисел. Однако $p$-базисная подгруппа такого прямого слагаемого, а следовательно, и группы $R(A)$ содержит свободное прямое слагаемое ранга большего, чем $m(0)$. Получаем противоречие со строением группы $B_{0}$.

Пусть теперь $p$-ранг $r(p, D)$ группы $D$ конечен. Тогда ясно, что $m(0)=1$. Далее, поскольку $D$ - периодическая делимая группа, то в ней можно выделить в качестве прямого слагаемого группу, изоморфную $\mathbb{Z}\left(p^{\infty}\right)$. Но тогда, учитывая, что $\operatorname{End}\left(\mathbb{Z}\left(p^{\infty}\right)\right) \cong \mathbb{J}_{p}$, получаем, что

$$
\operatorname{End}(D) \cong \mathbb{J}_{p} \oplus H_{2}
$$

А так как имеет место изоморфизм $\operatorname{End}(A) \cong A$, то и в редуцированной части $R(A)$ группы $A$ в качестве прямого слагаемого можно выделить подгруппу, изоморфную $\mathbb{J}_{p}$ :

$$
R(A) \cong \mathbb{J}_{p} \oplus R_{1}
$$


Но тогда, так как $\operatorname{End}\left(\mathbb{J}_{p}\right) \cong \mathbb{J}_{p}$, получаем

$$
\operatorname{End}(R(A)) \cong \mathbb{J}_{p} \oplus H_{3}
$$

Теперь

$$
A \cong \operatorname{End}(A) \cong \mathbb{J}_{p} \oplus H_{1} \oplus \operatorname{Hom}(R(A), D) \oplus \mathbb{J}_{p} \oplus H_{3} .
$$

Откуда, учитывая, что базисной подгруппой группы $\mathbb{J}_{p} \oplus \mathbb{J}_{p}$ является свободная группа ранга 2 , снова получаем противоречие с равенством $m(0)=1$.

Пусть теперь $A$ - редуцированная алгебраически компактная группа. В этом случае, согласно [1; с. 196], группа имеет вид

$$
A=\prod_{p \in P(A)} A p
$$

где $A p$ - p-адические компоненты группы $A, P(A) \subseteq \mathbb{P}$.

Теорема 4. Редуиированная алгебраически компактная группа $A=\prod_{p \in P(A)} A_{p}$ изоморфна своей группе эндоморфизмов $\operatorname{End}(A)$ тогда и толъко тогда, когда для любого простого р из $P(A) \quad A_{p} \cong \mathbb{J}_{p}$ или $A_{p} \cong \mathbb{Z}\left(p^{k}\right), k=k(p) \in \mathbb{N}$.

ДокАЗАТЕЛЬСтво. Пусть

$$
A=\prod_{p \in P(A)} A p
$$

- редуцированная алгебраически компактная группа. Согласно [1; с. 215] ее группа эндоморфизмов имеет вид

$$
\operatorname{End}(A) \cong \prod_{p \in P(A)} \operatorname{End}(A p),
$$

где каждая группа $\operatorname{End}(A p)$ является $p$-адической алгебраически компактной группой $[1 ;$ с. 236]. Таким образом, группы $A$ и $\operatorname{End}(A)$ изоморфны тогда и только тогда, когда для любого простого $p \in P(A)$ изоморфны группы $A p$ и $\operatorname{End}(A p)$. Покажем, что изоморфизм $A p \cong \operatorname{End}(A p)$ имеет место в том и только том случае, когда выполнены условия теоремы.

Достаточность. Пусть редуцированная алгебраически компактная группа $A=$ $\prod_{p \in P(A)} A p$ удовлетворяет условиям теоремы. Покажем, что для любого простого $p$ из $P(A)$ имеет место изоморфизм $A p \cong \operatorname{End}(A p)$.

Действительно, если $A p \cong \mathbb{J}_{p}$, то

$$
\operatorname{End}(A p) \cong \operatorname{End}\left(\mathbb{J}_{p}\right) \cong \mathbb{J}_{p} \cong A p .
$$

Если же $A p \cong \mathbb{Z}\left(p^{k}\right), k=k(p) \in \mathbb{N}$, то

$$
\operatorname{End}(A) \cong \operatorname{End}\left(\mathbb{Z}\left(p^{k}\right)\right) \cong \mathbb{Z}\left(p^{k}\right) \cong A p .
$$

Необходимость. Пусть для любого простого $p$ из $P(A)$ имеет место изоморфизм $A p \cong \operatorname{End}(A p)$. Покажем, что в этом случае редуцированная алгебраически компактная группа $A=\prod_{p \in P(A)} A p$ удовлетворяет условиям теоремы. 
Пусть $B=\bigoplus_{n \in N^{*}} B_{n}-p$-базисная подгруппа группы $A p$, где

$$
B_{0}=\bigoplus_{m(0)} \mathbb{Z}, \quad B_{n}=\bigoplus_{m(n)} \mathbb{Z}\left(p^{n}\right),
$$

$m(0)$ и $m(n)$ - некоторые кардиналы, $n \in \mathbb{N}$. Тогда в силу [1; с. 237] имеет место изоморфизм

$$
\operatorname{End}(A p) \cong \operatorname{Hom}(B, A p)
$$

Тогда

$$
\begin{aligned}
A p & \cong \operatorname{End}(A p) \cong \operatorname{Hom}\left(\bigoplus_{m(0)} \mathbb{Z}, A p\right) \oplus \operatorname{Hom}\left(\bigoplus_{n \in \mathbb{N}} B_{n}, A p\right) \\
& \cong \prod_{m(0)} \operatorname{Hom}(\mathbb{Z}, A p) \oplus \prod_{n \in \mathbb{N}} \operatorname{Hom}\left(\bigoplus_{m(n)} \mathbb{Z}\left(p^{n}\right), A p\right) \\
& \cong \prod_{m(0)} A p \oplus \prod_{n \in \mathbb{N}} \prod_{m(n)} \operatorname{Hom}\left(\mathbb{Z}\left(p^{n}\right), A p\right) .
\end{aligned}
$$

Ясно, что $m(0)$ - конечный кардинал (иначе $\left|\prod_{m(0)} A p\right|>|A p|$ ).

Таким образом, группа $A p$ имеет вид

$$
A p \cong \bigoplus_{m(0)} \mathbb{I}_{p} \oplus B^{*} \cong \prod_{m(0)} A p \oplus \prod_{n \in \mathbb{N}} \prod_{m(n)} \operatorname{Hom}\left(\mathbb{Z}\left(p^{n}\right), A p\right),
$$

где $B^{*}$ - редуцированная алгебраически компактная группа, $p$-базисная подгруппа которой не содержит свободных прямых слагаемых. Тогда

$$
\begin{aligned}
\operatorname{End}(A p) & \cong \bigoplus_{m(0)} \bigoplus_{m(0)} \mathbb{J}_{p} \oplus \operatorname{End}\left(B^{*}\right) \oplus \operatorname{Hom}\left(B^{*}, \bigoplus_{m(0)} \mathbb{J}_{p}\right) \oplus \operatorname{Hom}\left(\bigoplus_{m(0)} \mathbb{J}_{p}, B^{*}\right) \\
& \cong A p \cong \bigoplus_{m(0)} \mathbb{J}_{p} \oplus B^{*} .
\end{aligned}
$$

Следовательно, $m(0) \leqslant 1$.

Если $m(0)=0$, то

$$
A p=B^{*} \cong \operatorname{End}\left(B^{*}\right)=\operatorname{End}(A p) .
$$

Рассмотрим $p$-базисную подгруппу группы $B^{*}$ :

$$
B=\bigoplus_{n \in \mathbb{N}^{\prime}} \bigoplus_{m(n)} \mathbb{Z}\left(p^{n}\right)
$$

где $\mathbb{N}^{\prime}$ - подмножество множества $\mathbb{N}$ натуральных чисел, $m(n)$ - некоторые ненулевые кардиналы, $n \in \mathbb{N}^{\prime}$. Тогда

$$
A p=B^{*} \cong \operatorname{End}\left(B^{*}\right)=\operatorname{Hom}\left(B^{*}, B^{*}\right) \cong \operatorname{Hom}\left(B, B^{*}\right) \cong \operatorname{End}(A p) .
$$

Далее [1; с. 163],

$$
\operatorname{Hom}\left(B, B^{*}\right) \cong \prod_{n \in \mathbb{N}^{\prime}} \prod_{m(n)} \operatorname{Hom}\left(\mathbb{Z}\left(p^{n}\right), B^{*}\right)=\prod_{n \in \mathbb{N}^{\prime}} \prod_{m(n)} \operatorname{Hom}\left(\mathbb{Z}\left(p^{n}\right), B_{1} \oplus \cdots \oplus B_{n} \oplus B^{*}\right) .
$$


Следовательно [5], группа $\operatorname{End}\left(B^{*}\right)$ содержит прямое слагаемое вида

$$
\prod_{n \in \mathbb{N}^{\prime}} \prod_{m(n)} \operatorname{Hom}\left(\mathbb{Z}\left(p^{n}\right), B_{1} \oplus \cdots \oplus B_{n}\right) \cong \prod_{n \in \mathbb{N}^{\prime}} \prod_{m(n)}\left(B_{1} \oplus \cdots \oplus B_{n}\right)
$$

для каждого $n \in \mathbb{N}^{\prime}$. Откуда получаем, что $m(n)=1$ и $\left|N^{\prime}\right|=1$, т.е.

$$
A p=B^{*} \cong \mathbb{Z}\left(p^{n}\right) .
$$

Если $m(0)=1$, то

$$
A p \cong \mathbb{J}_{p} \oplus B^{*} \cong \operatorname{End}(A p) \cong \operatorname{Hom}\left(\mathbb{J}_{p}, B^{*}\right) \oplus \operatorname{End}\left(\mathbb{J}_{p}\right) \oplus \operatorname{End}\left(B^{*}\right) \oplus \operatorname{Hom}\left(B^{*}, \mathbb{J}_{p}\right) .
$$

Следовательно, группа $\operatorname{End}\left(B^{*}\right)$ изоморфна прямому слагаемому группы $B^{*}[1$; с. 200] и, следовательно, рассуждая как в предыдущем случае, получаем $B^{*} \cong \mathbb{Z}\left(p^{n}\right)$. Однако [1; с. 237], учитывая, что

$$
\operatorname{Hom}\left(\mathbb{J}_{p}, B^{*}\right) \cong \operatorname{Hom}\left(\mathbb{Z}, B^{*}\right) \cong B^{*},
$$

получим противоречие с изоморфизмом $A p \cong \operatorname{End}(A p)$. Таким образом, $B^{*}$ нулевая, и, так как $m(0)=1$, то $A p \cong \mathbb{J}_{p}$.

Ясно, что если группа $A$ изоморфна своей группе эндоморфизмов $\operatorname{End}(A)$, то и сама группа $\operatorname{End}(A)$ обладает этим же свойством. Обратная импликация, как будет показано ниже, не всегда имеет место. Группу $A$ будем называть End-грynnoü, если имеет место следующая импликация:

$$
\operatorname{End}(\operatorname{End}(A)) \cong \operatorname{End}(A) \Longrightarrow \operatorname{End}(A) \cong A .
$$

Теорема 5. Периодическая группа $A$ не является End-группой тогда и только тогда, когда либо существует р-компонента группы $A$, изоморфная группе $\mathbb{Z}\left(p^{\infty}\right)$, либо группа $A$ изоморна группе $\bigoplus_{p \in S(A)} \mathbb{Z}\left(p^{k}\right), k=k(p) \in \mathbb{N}$, где $S(A)$ - любое бесконечное подмножество множества $\mathbb{P}$.

ДокАЗАтЕЛЬство. Пусть $A$ - периодическая группа, для которой имеет место изоморфизм $\operatorname{End}(\operatorname{End}(A)) \cong \operatorname{End}(A)$. Группа $A$ периодическая, поэтому ее можно разложить в прямую сумму примарных групп, относящихся к различным простым числам, и тогда [1; с. 214]

$$
\operatorname{End}(A)=\operatorname{End}\left(\bigoplus_{p \in S(A)} A p\right) \cong \prod_{p \in S(A)} \operatorname{End}(A p) .
$$

С другой стороны, так как $A$ периодическая, то $\operatorname{End}(A)$ - редуцированная алгебраически компактная группа и поэтому согласно теореме 4

$$
\operatorname{End}(A p) \cong \mathbb{J}_{p}
$$

или

$$
\operatorname{End}(A p) \cong \mathbb{Z}\left(p^{k}\right), \quad k=k(p) \in \mathbb{N} .
$$

Поскольку $A p$ - периодическая группа, то в первом случае имеем $A p \cong \mathbb{Z}\left(p^{\infty}\right)$ и соответственно во втором случае $-A p \cong \mathbb{Z}\left(p^{k}\right)$. 
Таким образом, для периодической группы $A=\bigoplus_{p \in S(A)} A p$ изоморфизм

$$
\operatorname{End}(\operatorname{End}(A)) \cong \operatorname{End}(A)
$$

имеет место тогда и только тогда, когда либо $A p \cong \mathbb{Z}\left(p^{\infty}\right)$, либо $A p \cong \mathbb{Z}\left(p^{k}\right)$.

Теперь осталось применить теорему 1 . Если какая-нибудь из $p$-компонент группы $A$ изоморфна группе $\mathbb{Z}\left(p^{\infty}\right)$, тогда группы $\operatorname{End}(A)$ и $A$ не изоморфны и, следовательно, $A$ - не End-группа. Если же $A \cong \bigoplus_{p \in S(A)} \mathbb{Z}\left(p^{k}\right)$, то $\operatorname{End}(A) \cong A$ тогда и только тогда, когда $S(A)$ - конечное множество.

Пусть теперь $A$ - делимая группа. Так как имеет место теорема 5, то, не теряя общности, можем рассматривать только непериодические делимые группы.

Теорема 6. Делимая непериодическая группа является End-группой.

ДокАЗАТЕЛЬСтво. Пусть $A$ - делимая группа без кручения, не изоморфная своей группе эндоморфизмов $\operatorname{End}(A)$, т.е. (теорема 2) $r(A)>1$. Тогда группа $\operatorname{End}(A)$ также делимая и без кручения [1; с. 213], причем $r(\operatorname{End}(A))>1$. Применяя теорему 2 для группы $\operatorname{End}(A)$, заключаем, что группы $\operatorname{End}(\operatorname{End}(A))$ и $\operatorname{End}(A)$ не изоморфны.

Пусть $A$ - смешанная группа, тогда по теореме 2 группы $\operatorname{End}(A)$ и $A$ не изоморфны. Покажем, что в этом случае изоморфизм $\operatorname{End}(\operatorname{End}(A)) \cong \operatorname{End}(A)$ не имеет места. Поскольку группа $A$ делимая, то

$$
A=D(A)=D f(A) \oplus D t(A)=D f \oplus D t .
$$

И тогда, учитывая, что $\operatorname{Hom}(D t, D f)=0$ [1; с. 213], получаем

$$
\operatorname{End}(A) \cong \operatorname{End}(D f) \oplus \operatorname{Hom}(D f, D t) \oplus \operatorname{End}(D t) .
$$

Слагаемые $\operatorname{End}(D f)$ и $\operatorname{Hom}(D f, D t)$ - ненулевые делимые группы без кручения [1; c. 213]. Таким образом, в делимой части группы $\operatorname{End}(A)$ можно выделить как прямое слагаемое группу без кручения ранга больше 1 . Но тогда по теореме 3 группы $\operatorname{End}(\operatorname{End}(A))$ и $\operatorname{End}(A)$ не изоморфны.

Пусть теперь $A$ - нередуцированная группа. В силу теоремы 5 интерес представляют лишь непериодические группы.

Теорема 7. Нередуиированная непериодическая группа является End-группой.

Доказательство. Пусть $A=D(A) \oplus R(A)$ и $D(A) \neq 0$. Учитывая теорему 6 , можно считать, что $R(A) \neq 0$. Тогда

$$
\operatorname{End}(A) \cong \operatorname{End}(D(A)) \oplus \operatorname{Hom}(R(A), D(A)) \oplus \operatorname{End}(R(A)) .
$$

Случай 1. Пусть $D f(A) \neq 0$, а $D t(A)=0$, т.е. группа $D(A)$ без кручения, тогда

$$
D(\operatorname{End}(A)) \cong \operatorname{End}(D(A)) \oplus \operatorname{Hom}(R(A), D(A)) .
$$

По теореме 3 изоморфизм $\operatorname{End}(\operatorname{End}(A)) \cong \operatorname{End}(A)$ имеет место тогда и только тогда, когда $\operatorname{End}(A) \cong \mathbb{Q} \oplus \mathbb{Z}(n)$. Таким образом,

$$
\operatorname{End}(D(A)) \cong \mathbb{Q}
$$




$$
\operatorname{Hom}(R(A), D(A))=0 .
$$

Следовательно, $D(A) \cong \mathbb{Q}, R(A) \cong \mathbb{Z}(n)$ и $\operatorname{End}(A) \cong A$.

Случай 2. Пусть $D(A)=D f(A) \oplus D t(A)$, где $D f=D f(A) \neq 0$ и $D t=D t(A) \neq 0$. Тогда имеем

$$
\operatorname{End}(A) \cong \operatorname{End}(D f) \oplus \operatorname{Hom}(D f, D t) \oplus \operatorname{End}(D t) \oplus \operatorname{Hom}(R(A), D(A)) \oplus \operatorname{End}(R(A)) .
$$

Так как $D f$ - делимая группа без кручения, то группы $\operatorname{End}(D f)$ и $\operatorname{Hom}(D f, D t)$ также делимые и без кручения [1; с. 213]. Тогда по теореме 3 из изоморфизма $\operatorname{End}(\operatorname{End}(A)) \cong \operatorname{End}(A)$ следует, что $\operatorname{End}(D f) \cong \mathbb{Q}$ и $\operatorname{Hom}(D f, D t)=0$. Откуда получаем, что $D t=0$ и группа $D(A)$ без кручения. Таким образом, случай 2 сводится к случаю 1.

Случай 3. Пусть $D f(A)=0$, а $D t(A) \neq 0$, т.е. группа $D(A)$ периодическая, а группа $R(A) \neq 0$, следовательно, непериодическая. Поэтому по теореме 3 группы $A$ и $\operatorname{End}(A)$ не изоморфны. Покажем, что и группы $\operatorname{End}(\operatorname{End}(A))$ и $\operatorname{End}(A)$ в этом случае также не изоморфны.

Действительно, так как ранг без кручения группы $R(A)$ отличен от нуля, то в силу [1; с. 233] группа $\operatorname{Hom}(R(A), D(A))$ и, следовательно, и группа $\operatorname{End}(A)$, содержит делимую подгруппу. Далее, так как $D(A)$ - периодическая делимая группа, то она содержит как прямое слагаемое некоторую группу $\mathbb{Z}\left(p^{\infty}\right)$. Но тогда, учитывая, что $\operatorname{End}\left(\mathbb{Z}\left(p^{\infty}\right)\right) \cong \mathbb{J}_{p}$, получаем, что группа $\operatorname{End}(A)$ - нередуцированная группа, содержащая в качестве прямого слагаемого группу $\mathbb{J}_{p}$, а поэтому (по теореме 3 ) не изоморфна своей группе эндоморфизмов.

\section{СПИСОК ЦИТИРОВАННОЙ ЛИТЕРАТУРЫ}

[1] Л. Фукс, Бесконечные абелевы группы, 1, Мир, М., 1974.

[2] L. Fuchs, Abelian Groups, Hungar. Acad. Sci., Budapest., 1966.

[3] А. М. Себельдин, "Группы гомоморфизмов вполне разложимых абелевых групп без кручения", Изв. вузов. Матем., 7 (1973), 77-84.

[4] А. М. Себельдин, "О группах гомоморфизмов абелевых групп без кручения", Группь и модули, Изд-во Томск. ун-та, Томск, 1976, 70-77.

[5] A. M. Sebeldin, "Isomorphisme naturel des groupes des homomorphismes des groupes abeliens", Ann. de l'IPGANC, 8A (1982), 155-158.

Е. М. Коленова, А. М. Себельдин

Нижегородский государственный педагогический институт

E-mail: kem17@yandex.ru, sebeldinam@rambler.ru
Поступило

08.10 .2004

Исправленный вариант

23.03.2006 